\title{
1E0657-56: A CONTENDER FOR THE HOTTEST KNOWN CLUSTER OF GALAXIES
}

\author{
W. Tucker ${ }^{1}$, P. Blanco, S. Rappoport \\ University of California, San Diego \\ Center for Astrophysics and Space Sciences \\ 9500 Gilman Drive, Code 0111 \\ La Jolla, CA 92093 \\ L. David, D. Fabricant, E.E. Falco, W. Forman \\ Harvard-Smithsonian Center for Astrophysics \\ 60 Garden Street \\ Cambridge, MA 02138
}

A. Dressler

Carnegie Observatories

813 Santa Barbara Street

Pasadena, CA 91101

M. Ramella

Osservatorio Astronomico di Trieste

via G.B. Tiepolo, 11,I-34131

Trieste, Italy

Received —

submitted to The Astrophysical Journal

\footnotetext{
${ }^{1}$ Also at the Harvard-Smithsonian Center for Astrophysics
} 


\begin{abstract}
We identify the extended Einstein IPC X-ray source, 1E0657-56, with a previously unknown cluster of galaxies at a redshift of $z=0.296$. Optical CCD images show the presence of a gravitational arc in this cluster and galaxy spectra yield a cluster velocity dispersion of $1213_{-191}^{+352} \mathrm{~km} \mathrm{~s}^{-1}$. X-ray data obtained with the ROSAT HRI and ASCA indicate that 1E0657-56 is a highly luminous cluster in which a merger of subclusters may be occurring. The temperature of the hot gas in $1 \mathrm{E} 0657-56$ is $\mathrm{kT}=17.4 \pm 2.5 \mathrm{keV}$, which makes it an unusually hot cluster, with important cosmological implications.
\end{abstract}

Subject headings: galaxies: clusters:individual (1E0657-56) - intergalactic medium - large-scale structure of the universe - X-rays: galaxies 


\section{INTRODUCTION}

Measurements of the gas temperature in clusters of galaxies is a potentially powerful tool for testing cosmological models since the gas temperature provides a direct measurement of the cluster mass. The cluster mass function is directly traceable to the underlying cosmology and can be used to determine the normalization and slope of the initial mass fluctuation spectrum in the early Universe (Peebles et al. 1989, Henry \& Arnaud 1991, Lilje 1992, Bartlett \& Silk 1993, White et al. 1993, Colafrancesco \& Vittorio 1994, Hattori et al. 1995, Eke et al. 1995, Eke et al. 1996, Pen 1996, Viana \& Liddle 1996, Oukbir et al. 1997, Oukbir \& Blanchard 1997, Bahcall et al. 1997). The usefulness of this method is limited by the uncertainty in the parameters used to fit the theory of cluster formation to the observed cluster temperature distribution function (Colafrancesco et al. 1997). Observations of very hot clusters can alleviate this problem to a certain extent. The cluster temperature function is predicted to have an exponential cutoff beyond a critical temperature which depends on the cosmological model, so the differences between the theoretical predictions increase exponentially beyond the critical temperature.

Here we report on optical and X-ray observations of a newly discovered cluster, 1E0657-56. The measured temperature $\mathrm{kT}=17.4 \pm 2.5 \mathrm{keV}$ makes it a strong candidate for the hottest known cluster. The interpretation of this temperature is complicated by evidence that the cluster is in the process of merging with a subcluster (see e.g. Schindler \& Muller, 1993, Roettiger et al 1997). Since the subcluster has a mass on the order of one-tenth the mass of the primary cluster, we argue that the high temperature is a true indication of a large primary mass. If this interpretation is correct, then the existence of such a cluster at the observed redshift $z=0.296$ is very difficult to reconcile with current theoretical models for a critical density $(\Omega=1)$ universe.

This letter is organized in the following manner. The optical and X-ray observations are 
presented and analyzed in $\oint 2$, and the implications of the exceptionally high temperature of the hot gas in 1E0657-56 are considered in $\oint 3$.

\section{OBSERVATIONS}

\subsection{Optical Observations}

The cluster 1E0657-56, was detected as an extended source on a scale of $3^{\prime}$ by the Imaging Proportional Counter (IPC) onboard the Einstein Observatory. The source remained unidentified until a CCD image of this source was obtained with the Las Campanas 2.5 meter telescope as part of a program to search for possible failed clusters

of galaxies, i.e., clusters with hot gas but no galaxies (Tucker, Tananbaum \& Remillard 1995). The CCD image revealed the presence of a rich cluster of galaxies and a luminous arc about 12 arcsec long. Follow-up imaging and spectroscopic observations with the ESO NTT telescope in December 1993 confirmed the presence of the arc and yielded a cluster redshift of $z=0.296$. The corresponding luminosity distance is $D=2.04 h_{50}^{-1} \mathrm{Gpc}$ for $q_{0}=\Lambda=0$, where $h_{50}$ is the Hubble constant in units of $50 \mathrm{~km} \mathrm{~s}^{-1} \mathrm{Mpc}^{-1}$.

We use our NTT spectroscopic data to calculate the cluster velocity dispersion. The method of Danese et al (1980) is used to include the effects of measurement errors. From the 24 measured redshifts we only include galaxies with velocities between $80,000 \mathrm{~km} \mathrm{~s}^{-1}$, and $100,000 \mathrm{~km} \mathrm{~s}^{-1}$, and within $R_{\max }=3$ arcmin of the brightest $\mathrm{cD}$ galaxy in the cluster. The resulting velocity dispersion is $\sigma_{v}=1213_{-191}^{+352} \mathrm{~km} \mathrm{~s}^{-1}$, based on 13 galaxies. When we vary $R_{\max }$ between $1 \operatorname{arcmin}$ and $4 \operatorname{arcmin}$, we find $\sigma_{v}$ varies from 830 to $1515 \mathrm{~km} \mathrm{~s}^{-1}$, respectively, and the corresponding number of galaxies varies from 7 to 15. At the lower limit, the number of galaxies is too small to yield useful errors; at the upper limit, the errors are comparable to those with $R_{\max }=3$ arcmin. 


\subsection{ROSAT HRI Observations}

The ROSAT HRI observed 1E0657-56 for a total live time of $57.7 \mathrm{ksec}$ between 8 February 1995 and 27 June 1995. A contour image of the background subtracted HRI image is shown in Figure 1. This figure shows that the X-ray emission is extended and double-peaked, indicating either a merger event or two spatially distinct groups. The X-ray flux of the secondary peak, which is also an extended source, comprises only $4 \%$ of the total flux.

The surface brightness profile of the primary cluster was obtained by extracting the net counts in concentric annuli about the primary X-ray peak after excluding the western quadrant (which contains the secondary peak) and all point sources. We then fit the observed X-ray surface brightness profile to the standard hydrostatic-isothermal $\beta$ model,

$$
\Sigma(R)=\Sigma_{0}\left(1+(R / a)^{2}\right)^{-3 \beta+1 / 2}
$$

with the addition of a flat background component. The best fit is obtained with a core radius of $a=62^{\prime \prime} \pm 10^{\prime \prime}$ (corresponding to $360 \pm 50 h_{50}^{-1} \mathrm{kpc}$ ), $\beta=0.62 \pm 0.07$, and a central

electron number density $n_{e}=7.5 \times 10^{-3} h_{50}^{1 / 2} \mathrm{~cm}^{-3}$. Integrating the best fit $\beta$ model gives a gas mass within $1 h_{50}^{-1} \mathrm{Mpc}$ of $2.0 \pm 0.3 \times 10^{14} h_{50}^{-5 / 2} M_{\odot}$. The net flux and luminosity of the primary cluster is then determined by multiplying the flux from the 3 quadrants centered on the primary peak by $4 / 3$ to account for the flux underlying the secondary peak. Using the gas temperature derived from the ASCA data gives $f_{0.1-2.4 \mathrm{keV}}=5.6 \pm 0.6 \times 10^{-12} \mathrm{erg}$ $\mathrm{cm}^{-2} \mathrm{~s}^{-1}$ and $L_{b o l}=1.4 \pm 0.3 \times 10^{46} h_{50}^{-2} \mathrm{erg} \mathrm{s}^{-1}$. 


\subsection{ASCA Observations}

Standard screening criteria were applied to the SIS and GIS data to exclude data taken during Earth occultations, periods of high particle flux, and the portions of ASCA orbit that pass through the South Atlantic Anomaly. Hot and flickering pixels were removed with SISCLEAN and GISCLEAN. The resulting screened exposure times are: SIS0 (20.7 ksec); SIS1 (20.0 ksec); GIS2 (21.7 ksec); and GIS3 (21.7 ksec). Source spectra were extracted using a rectangular region of $4.2^{\prime}$ by $3.7^{\prime}$ for the SIS0 data, a rectangular region of $4.2^{\prime}$ by $3.0^{\prime}$ for the SIS1 data, and circular regions of radii $6.4^{\prime}$ and $7.1^{\prime}$ for the GIS2 and GIS3 data, respectively. Background spectra were taken from available blank sky observations.

All four background subtracted spectra are then simultaneously fit to a singletemperature Raymond thermal plasma model. The best fit model $\left(\chi^{2}=692\right.$ for 778 spectral bins) and residuals are shown in Figure 2a. The best fit model has a temperature of $\mathrm{kT}=17.4 \pm 2.5 \mathrm{keV}$ (90\% confidence level) and an Fe abundance of $0.35 \pm 0.15$ solar $\left(\mathrm{Fe} / \mathrm{H}=4.68 \times 10^{-5}\right)$. A red-shifted $6.9 \mathrm{keV}$ line, corresponding to the FeXXVI $K_{\alpha}$ line is also detected, but only at the $2.5 \sigma$ level. Figure $2 \mathrm{~b}$ shows a comparison of the SIS0 data from 1E0657-56 with the best fit single-temperature Raymond model for A2163 $(\mathrm{kT}=12 \pm 2 \mathrm{keV}$; Markevitch et al 1996). 1E0657-56 is clearly hotter than A2163. The best fit temperature is also greater than that of MS1054-53, reported to have a temperature of $14.7_{-3.5}^{+4.6} \mathrm{keV}$ (Donahue et al 1997). At $\mathrm{kT}=17.4 \mathrm{keV}, 1 \mathrm{E} 0657-56$ is to our knowledge the hottest known cluster of galaxies. For a temperature of $17 \mathrm{keV}$ and the velocity dispersion

given above, we find $\beta_{\text {spec }}=\mu m_{p} \sigma_{v}^{2} / k T=0.56$, which agrees well with the value of $\beta$ determined from the fit to the HRIHRI surface brightness profile.

An absorbed power-law model (photon index $=1.5 \pm 0.05$ ) also provides an acceptable fit to the ASCA data, with a reduced chi-squared of 0.932. However the ROSAT HRI image shows that 1E0657-56 is extended in the 0.1 to $2.4 \mathrm{keV}$ band, so a pure power law 
from an active galactic nucleus (AGN) cannot explain the entire 0.5 to $10 \mathrm{keV}$ ASCA spectrum. The FWHM of the ASCA/SIS point response function is comparable to the extent of the cluster, so we cannot determine if the hard emission outside of the PSPC bandpass is extended. However, the centroids of the ASCA image in both the 1-4 keV and 4-10 keV bands are coincident with the centroid of the ROSAT image, supporting the assumption that the ROSAT and ASCA emission have the same origin, namely the hot cluster gas. Assuming the gas is isothermal gives a total gravitating mass within $1 h_{50}^{-1}$ Mpc of $1.1 \pm 0.2 \times 10^{15} h_{50}^{-1} M_{\odot}$, based on the equation of hydrostatic equilibrium, the best fit $\beta$ model, and the ASCA determined gas temperature. This gives a gas mass fraction of $0.18 h_{50}^{-3 / 2}$, consistent with other rich clusters (David, Jones, \& Forman 1995; White \& Fabian 1995).

\section{DISCUSSION}

The thermal energy content of the cluster gas within a radius of $1 h_{50}^{-1} \mathrm{Mpc}$ is approximately $2 \times 10^{64}$ ergs. It is difficult to account for this energy content by any process other than gravitational collapse. Assuming a thermal energy input of $10^{51}$ ergs per supernova, $1.5 \times 10^{13}$ supernovae would be required to heat the gas. Assuming an equal mixture of Type Ia supernovae (Fe yield of $0.6 M_{\odot}$ ) and Type II supernovae (Fe yield of $\left.0.08 M_{\odot}\right)$, the resulting iron abundance would be more than ten times the solar abundance and thirty times greater than implied by the ASCA data. A quasar can generate $\sim 10^{46}$ ergs $\mathrm{s}^{-1}$ of energy for $\sim 10^{9}$ years; ten such quasars would be required to explain the thermal energy content of 1E0657-56, but then the space density of quasars in the cluster would have to be $\sim 100$ times greater than the cosmic average.

The cluster is observed to be a radio source; Griffith and Wright (1993) detected a $1.7 \mathrm{mJy}$ radio source at $4850 \mathrm{MHz}$. Inverse Compton scattering of the microwave background 
off the relativistic electrons in the radio source could produce an extended, hard X-ray source. The intensity of this source in the $0.1-10 \mathrm{keV}$ band is of the order of

$$
\begin{aligned}
L_{C}(0.1-10 \mathrm{keV}) & \sim L_{\text {syn }}\left(8 \pi w_{b b} / B^{2}\right)\left(\nu_{x} / \nu_{b b} \gamma^{2}\right) \\
& \sim 6 \times 10^{39}\left(10^{-6} \text { gauss } / \mathrm{B}\right) \mathrm{erg} \mathrm{s}^{-1}
\end{aligned}
$$

However, since the equipartition magnetic field is $B_{e q} \sim 10^{-6}$ gauss, the inverse Compton contribution to the $\mathrm{X}$-ray intensity is negligible.

Numerical simulations of merging clusters (e.g. Roettiger, et al. 1997, Evrard et al. 1996, Schindler \& Muller, 1993) show that temperatures of order $20 \mathrm{keV}$ can be achieved in mergers, a conclusion confirmed by the ASCA observations of Abell 754 (Henriksen \& Markevitch 1996). However, the simulations and the observations both indicate that for mergers with high cluster/subcluster mass ratio, the high temperature regions are usually localized, and do not affect significantly the average cluster temperature. In A754, the average temperature is $\sim 9 \mathrm{keV}$, even though a temperature in the range $>12 \mathrm{keV}$ is found in one region (Henriksen \& Markevitch 1996). This is in agreement with the numerical simulations of Roettiger et al.( 1997) for a cluster/subcluster mass ratio of 8:1. Their calculations show little change of the emission weighted temperature, except for a peak at the time of the merger which is confined to the central $300 \mathrm{kpc}$ for roughly a few hundred million years. For 1E0657-56, we can estimate the mass ratio from the HRI observation, which indicates that $4 \%$ of the flux comes from the secondary peak which occupies $10 \%$ of the volume, $V$. Since the luminosity $L \propto M^{2} / V$, the mass ratio is $\sim 16: 1$, so the effect of the merger on our emission weighted temperature should not be large for this particular cluster.

One of the strongest correlations between the X-ray properties of clusters of galaxies 
is that between the gas temperature and X-ray luminosity (David et al. 1993; Mushotzky \& Scharf 1997). Figure 3 shows that, within the errors, the temperature of 1E0657-56 is consistent with this relation, providing further support that the high temperature reflects a global property of the cluster, and is not due to a small scale temperature enhancement.

In summary, the numerical simulations do illustrate the importance of having spatial as well as spectral information before using cluster temperatures as an indicator of virial masses, but in the present case we conclude that the unusually high temperature of 1E0657 is due to an unusually deep potential well and hence a large virial mass.

Every cluster that is discovered with a temperature greater than about $15 \mathrm{keV}$ places an additional straw on the "camel's back" of cosmological theories for the formation of clusters of galaxies that require $\Omega=1$. The standard normalization for primordial density fluctuations is derived from the number density of 5-8 keV clusters (Henry \& Arnaud 1991, Eke et al 1996, Pen 1996, Viana \& Liddle 1996). Differences between cosmological models are strongly amplified at temperatures of $17 \mathrm{keV}$. For example, the expected number of clusters with a temperature greater than $17.4 \mathrm{keV}$, in a volume bounded by a redshift $z=0.3$ is $N(T>17.4, z<0.3)=0.3$ for $\Omega=0.37$ and $N(T>17.4, z<0.3)=4 \times 10^{-6}$ for $\Omega=1.00$ for a CDM spectrum normalized to fit the cluster number density at 5.5 $\mathrm{keV}$ (U. Pen, private communication). The discovery of just one high temperature cluster thus effectively rules out models with a cold dark matter (CDM) type of power spectrum of fluctuations and $\Omega=1$. Other authors (Donahue et al. 1997, Luppino \& Gioia 1995, Ostriker 1993, and references cited therein) have noted that standard CDM models with $\Omega=1$ cannot explain the COBE measurements of CMBR temperature anisotropies (Smoot et al 1992), as well as the abundances of clusters and galaxies.

In the future we can anticipate that the discovery and study of very hot gas in high redshift clusters of galaxies with ASCA and future observatories such as AXAF and XMM 
will provide severe tests for cosmological models for the formation of large-scale structure in the universe.

We are grateful for the assistance of K. Arnaud, P. Gorenstein, U. Pen, and R. Mushotzky. This research was supported by NASA Contract NAS8-39073 and the Smithsonian Institution. We benefited from the use of the NASA Extragalactic Data Base to locate the radio source identified with 1E0657-58. 


\section{REFERENCES}

Bahcall, N., Fan, X. and Cen, R. 1997 Ap.J.485, L53.

Bartlett, J. \& Silk, J. 1993 Ap.J. 407, L45.

Colafrancesco, S. \& Vittorio, N. 1994 Ap.J. 422, 443.

Colafrancesco, S., Mazzotta, P., \& Vittorio, N. 1997 Ap. J. 488, 566

Danese,L. De Zotti, G.\& Di Tullio,G. 1980 A\&A 82,322

David, L., Jones, C., Forman, W., Vrtilek, S., and Arnaud, K. A 1993 Ap.J. 412, 479

David, L., Jones, \& Forman, W. 1995, Ap.J., 445, 578.

Donahue, M., Gioia, I., Luppino, G., Hughes, J., and Stocke, J. Ap. J. (submitted, 1997)

Elbaz, D., Arnaud, M. and Bohringer, H.1995 A\&A 293, 337

Eke, V., Cole, S. and Frenk, C. 1996 M.N.R.A.S. 282,263

Eke, Luppino, G. \& Gioia, I. 1995 Ap. J. 445, L77

Griffith, M. and Wright, A. 1993 A.J. 105,1666

Hattori, M. \& Matsuzawa, H. 1995 A \& A, 300, 637

Henry, P. \& Arnaud, K. 1991 Ap. J. 372, 410

Lilje, P. 1992 Ap.J. 386, L33

Markevitch, M., Mushotzky, R., Inoue, H., Yamashita, K., Furuzawa, A., Tawara, Y. 1996 Ap.J. 456, 437

Mushotzky, \& R.Scharf. C. 1997 Ap. J. 482, L13

Ostriker, J. 1993, ARA\&A 31, 689

Oukbir, J. \& Blanchard, A. 1997 Astron. Astrophys. 317,1

Oukbir, J. , Bartlett, J. \& Blanchard, A. 320, 365 
Peebles, J., Daly, R. \& Juszkiewicz, R. 1989 Ap. J. 347, 563

Pen, U. 1996 astro-ph/9610147

Roettiger, K., Burns, J. \& Loken, C. 1996 Ap. J. 473, 651

Roettiger, K. Loken, C. \& Burns, J. 1997 Ap. J. Suppl. 109, 307

Schindler, S. \& Muller, E. 1993 Astron. Astrophys. 272, 137

Smoot, G. et al 1992 Ap.J. 396, L1

Tanaka, Y. Inoue, H. and Holt, S.S. 1994 PASJ 46, L37

Tsujimoto, T., Nomoto, K., Yoshi, Y., Hashimoto, M., Yanagida, S. and Thielemann, F. 1995 MN 277, 945

Tucker, W. Tananbaum, H. and Remillard, R. 1995 Ap.J.444, 532

Viana, P. and Liddle, A. 1996 MNRAS 281, 323

Vikhlinin, A., Forman, W., and Jones, C. 1994, ApJ., 435, 162.

White, S., Efstathiou, G. \& Frenk, C. 1993,, MNRAS 262, 1023

White, D. \& Fabian, A. 1995, MNRAS, 273, 72.

This manuscript was prepared with the AAS LATEX macros v4.0. 
Fig. 1.- Contour map of the ROSAT HRI image for 1E0657-56. The contour map is generated from a background subtracted, wavelet smoothed image using the technique described in Vikhlinin, Forman, and Jones (1994). The peak contour level is drawn at a surface brightness of $5.9 \times 10^{-2}$ cts $\operatorname{arcmin}^{-2} \mathrm{sec}^{-1}$. The remaining contour levels are spaced at factors of two decrease in surface brightness. The lowest contour is $4 \sigma$ above background.

Fig. 2.- a) Best fit Raymond thermal plasma model to the ASCA data. Filled squares (SIS), Open squares (GIS). b) Comparison of the SIS0 spectrum of 1E0657-56 with the best fit spectrum for A2163 (solid line). The 1E0657-56 data points above $6 \mathrm{keV}$ diverge noticeably from the A2163 fit, confirming that 1E0657-56 is hotter than A2163.

Fig. 3.- The luminosity-temperature plot for clusters of galaxies, adapted from David et al.1993 and Mushotzky \& Scharf 1997, showing the location of A2163, MS1054-53 and 1E 0657-56. 


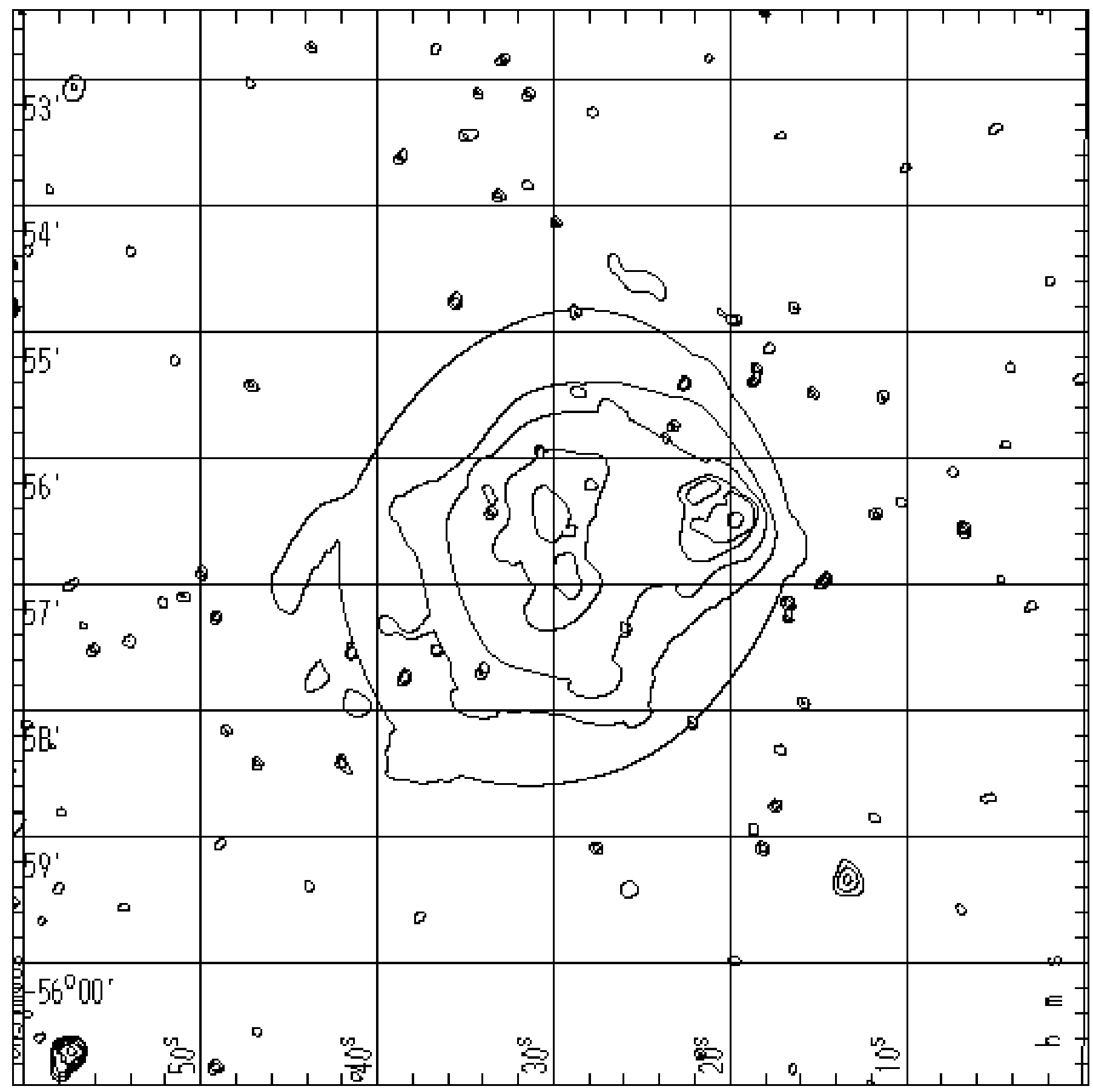




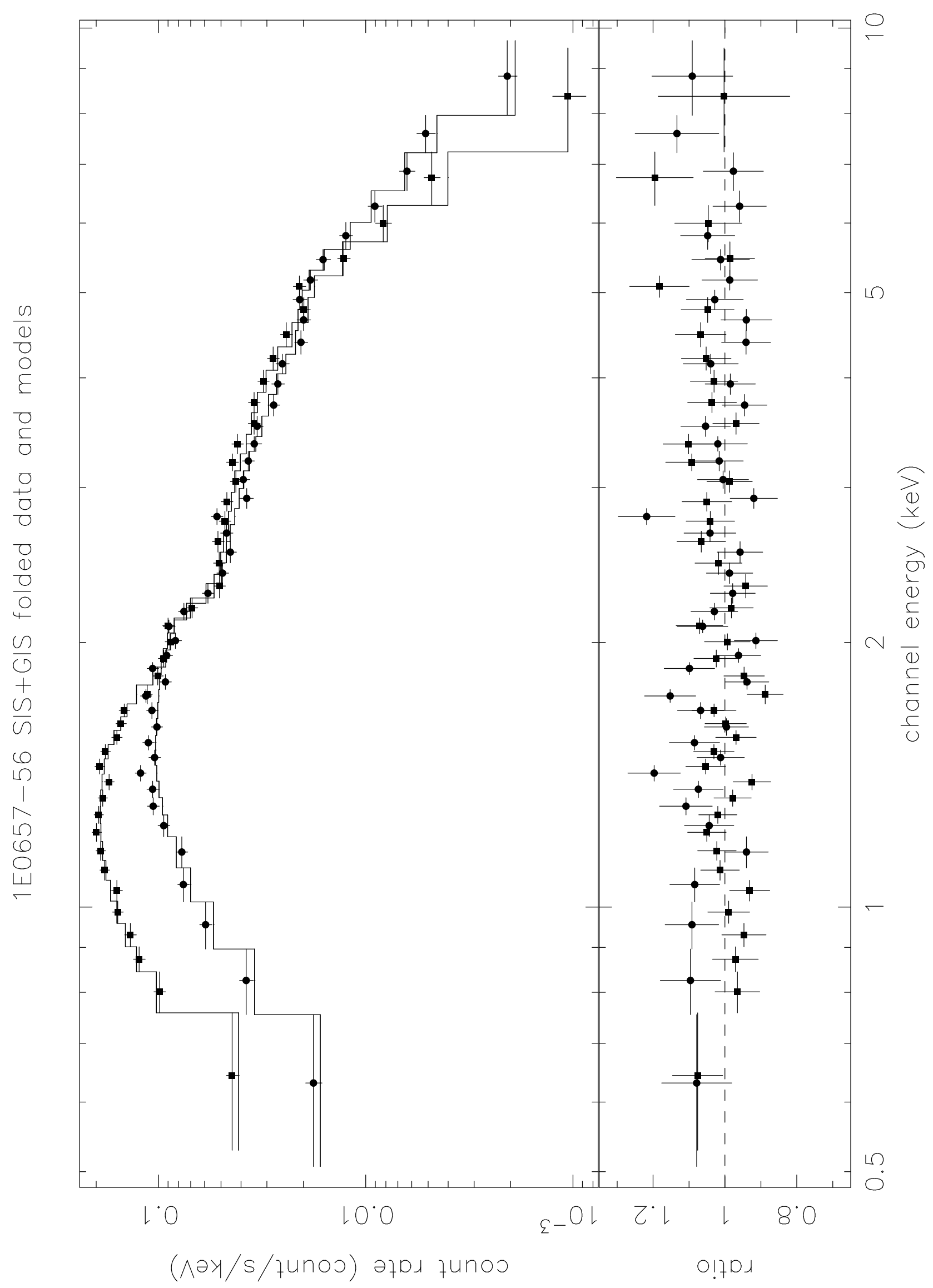




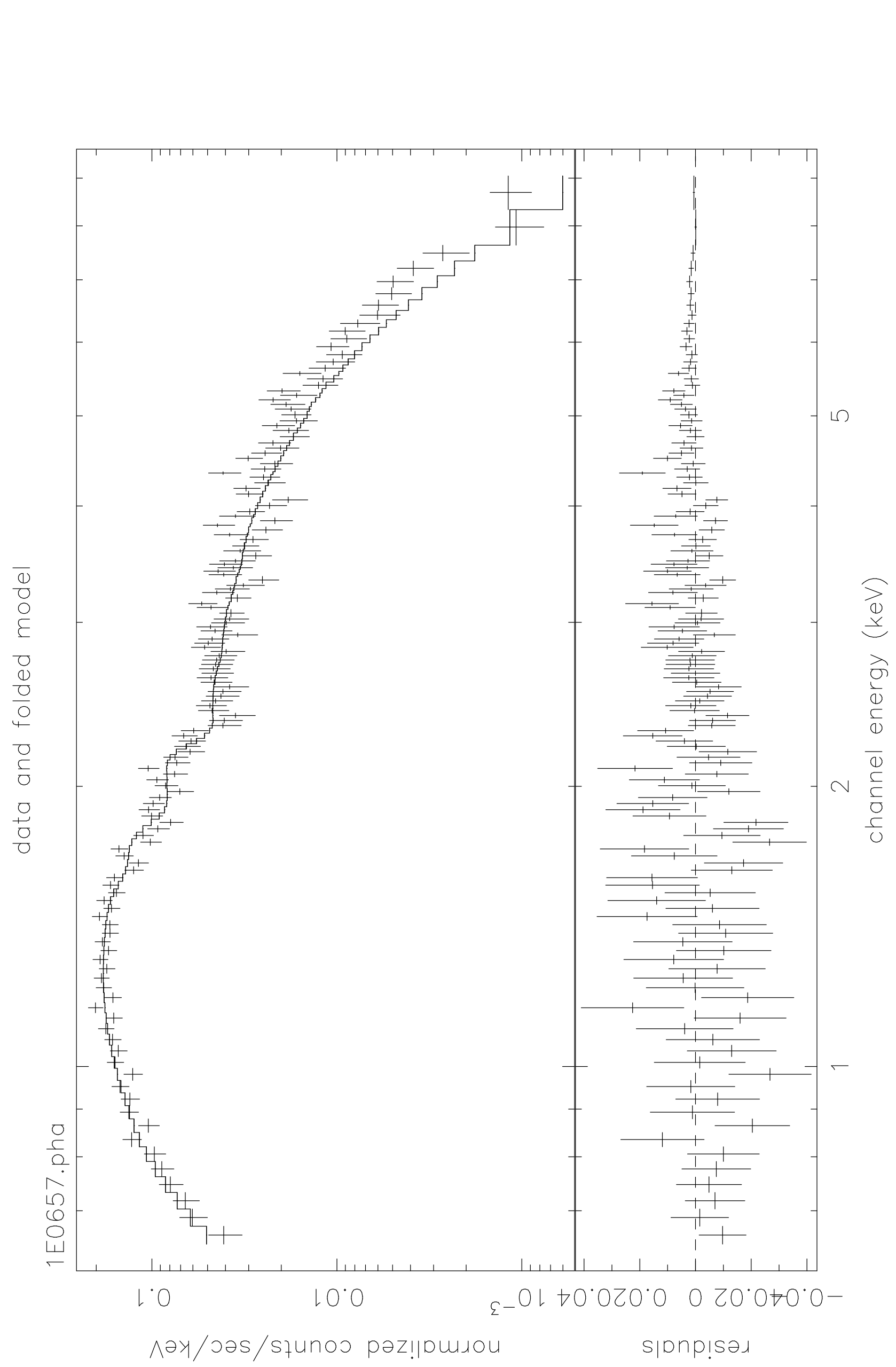




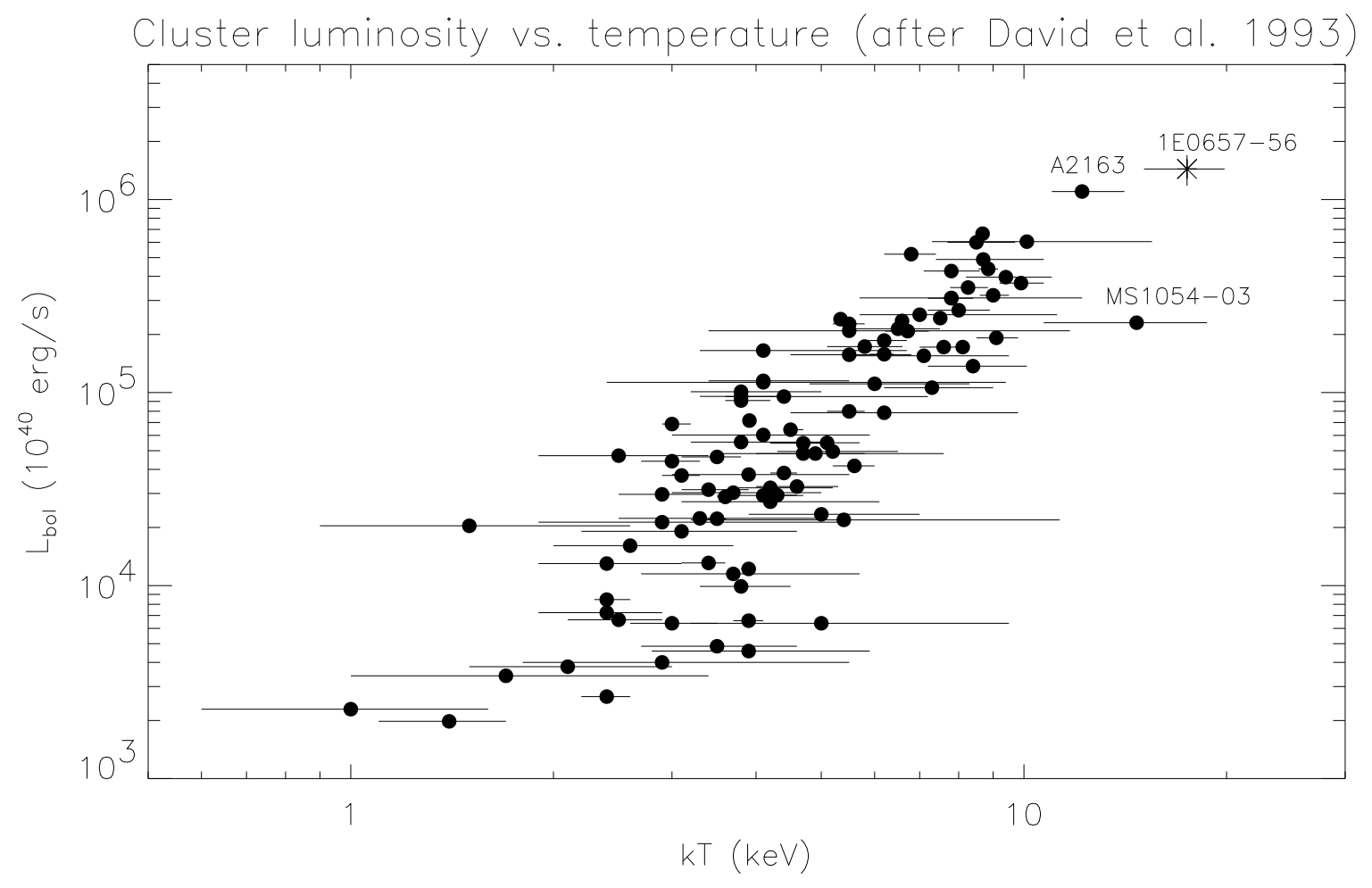

